\title{
Electronic structure calculations of metal-nanotube contacts with or without oxygen adsorption
}

\author{
Noejung Park ${ }^{1, *}$ and Suklyun Hong ${ }^{2, \dagger}$ \\ ${ }^{1}$ Department of Applied Physics, Dankook University, Seoul 140-714, Korea \\ ${ }^{2}$ Department of Physics and Institute of Fundamental Physics, Sejong University, Seoul 143-747, Korea
}

(Received 2 February 2005; published 6 July 2005)

\begin{abstract}
The Schottky barrier at the metal-nanotube contact has been a prime issue in the nanoscale devices. Here we use $a b$ initio density-functional calculations to investigate the electronic structure and the Fermi level alignment at the metal-nanotube contacts. Consistent with the common concept of the large (small) work function of gold (aluminum) surfaces, the Fermi level of the gold layer is found to be aligned at the valence band edge, while that of the aluminum sits at the conduction band edge of the semiconducting carbon nanotube. However, upon the oxidation, the work function of aluminum surface becomes as large as that of the clean gold surface, causing the Fermi level to be aligned at the valence band edge of the semiconducting nanotube. This suggests that the carrier type of the nanotube field effect transistor could transform from $n$-type to $p$-type upon oxygen adsorption on the electrode surface. The oxidation-induced increase of the tunneling barrier is also investigated.
\end{abstract}

DOI: $10.1103 /$ PhysRevB.72.045408

PACS number(s): 81.07.De, 73.22.-f, 85.35.Kt

\section{INTRODUCTION}

The carbon nanotube (CNT) has been considered as the most promising building block for future electronic devices because of its unique one-dimensional geometry, mechanical, and chemical robustness, and excellent transport property. ${ }^{1,2}$ The unprecedented high mobility, large current density, and a delicate response to the gate bias have been demonstrated in the CNT field effect transistor (FET) ${ }^{3-5}$ Natural research trends nowadays are directed toward the integration skills of the individual transistor ${ }^{6}$ and the design of its transport character. Transistors with either $n$ - or $p$-type behavior operating under the same voltage range would be basic constituting units for the nanotube-based CMOS technology. Amidst various trials to dope the carbon nanotube with the desired carrier type, IBM group and a few others have demonstrated that the Schottky barrier at the metal-CNT contact could be adjusted, electrostatically as well as chemically, to achieve the desired carrier type in the CNT-FET. ${ }^{7-11}$

The effect of oxygen adsorption on CNT-FET has been discussed in two different view points. Common observations of the $p$-type behaviors of the nanotube devices have been attributed to the doping by the adsorbed oxygen from the ambient air. ${ }^{12-14}$ Meanwhile, Derycke et al. have suggested the main effect of the oxygen adsorption lies in the adjustment of the Schottky barrier rather than the bulk doping effect of the CNT itself. ${ }^{10,11}$ Subsequent experimental and theoretical studies supported this view, showing a negligible charge transfer between oxygen adsorbate and the nanotube wall. ${ }^{15-17}$ Nevertheless, the bulk doping effect would be obviously effective for networks of CNTs or a very long individual nanotube. ${ }^{12,13}$ However, as the channel length of the current silicon devices approaches several tens nanometers, the future technology would employ a single and short nanotube for a further miniaturized device. Thus the effect of oxygen adsorption on the Schottky barrier should be reviewed as a more relevant context for a future nanoelectronics. ${ }^{10,11}$

In this work, we use the $a b$ initio density-functional method to investigate the electronic structure at the metalnanotube contact and the effect of oxygen adsorption on it. We consider gold $(\mathrm{Au})$ and aluminum (Al) as typical examples of high and low work function metals, respectively. We find that the Fermi level of aluminum is aligned at the conduction band edge, while that of gold sits at the valence band edge of the carbon nanotube. Upon the oxidation, the increment of the aluminum work function is found to be about $1 \mathrm{eV}$. Considering the practical band gap of the semiconducting carbon nanotube, this change in the metal work function could have a substantial influence on the Schottky barrier between the electrode and the semiconducting nanotube. The effect of the oxygen adsorption on the tunneling barrier between metal and nanotube is also investigated.

\section{COMPUTATIONAL METHOD}

To investigate electronic structure and the Fermi level alignment at the metal-nanotube contacts, we have performed $a b$ initio calculations using the Vienna $a b$ initio simulation package (VASP). ${ }^{18,19}$ For exchange and correlation, the PBE-type functional is used in the generalized gradient approximation (GGA) ${ }^{20}$ Electron-ion interactions are described using the projector augmented wave (PAW) method, ${ }^{21}$ and the plane-wave basis with the kinetic energy cutoff of $400 \mathrm{eV}$ is employed to describe the Kohn-Sham single-electron equations. A Gaussian broadening with a width of $0.05 \mathrm{eV}$ is used to accelerate the convergence in the $k$-point sum. Atoms are relaxed with a residual force smaller than $0.02 \mathrm{eV} / \AA$.

\section{RESULTS AND DISCUSSION}

In order to investigate the Schottky barrier at the metalnanotube contacts we locate the semiconducting $(10,0)$ nanotube on metal surfaces. From the bulk fcc structures, three layers of $\mathrm{Al}(\mathrm{Au})$ are excised to form a two-dimensional slab geometry, as shown in Figs. 1(a) and 1(b). The unit cell 

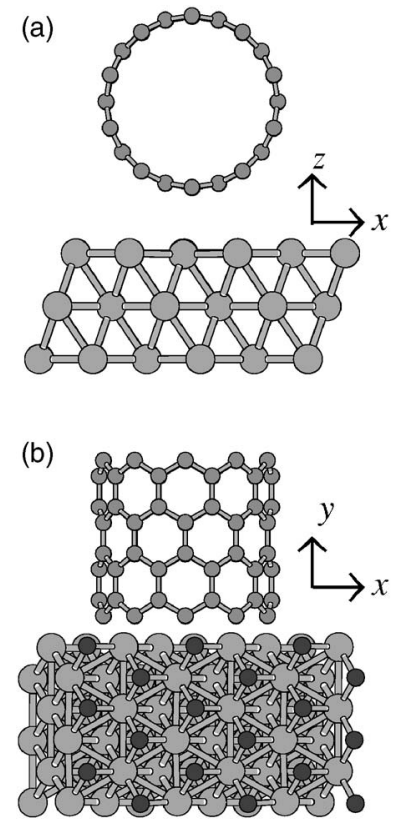
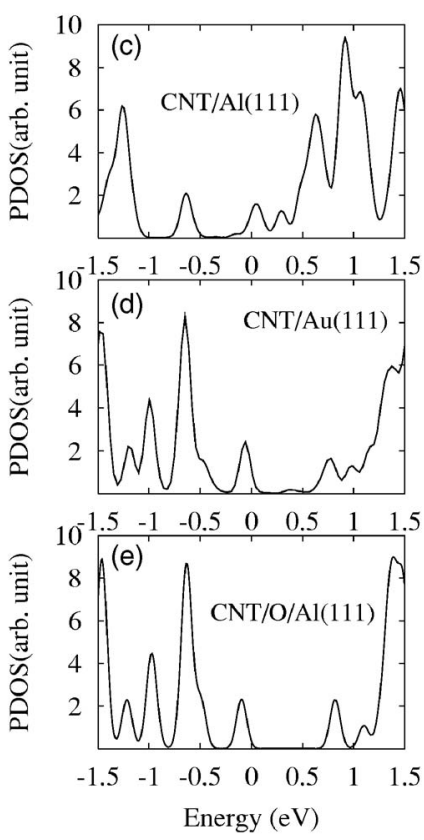

FIG. 1. (a) A cross-sectional side view of the optimized geometry of the $(10,0)$ nanotube on the $\mathrm{Au}(111)$ surface, and (b) the top view of the $(10,0)$ nanotube on the oxidized $\mathrm{Al}(111)$ surface. For visual clarity, the nanotube section in (b) is shifted along the axial direction to avoid the overlap. Larger gray spheres in (a) and (b) represent the aluminum and gold atoms, while smaller gray and dark spheres correspond to the carbon and oxygen atoms, respectively. Projected densities of states for the $(10,0)$ carbon nanotube in contact with (c) the $\mathrm{Al}(111)$ surface, (d) the $\mathrm{Au}(111)$ surface, and (e) the oxidated $\mathrm{Al}(111)$ surface, respectively. In (c), (d), and (e), the Fermi levels are set to zero.

along the axial direction $(y)$ of the nanotube is twice the minimal unit cell of the $(10,0)$ nanotube $(\approx 8.49 \AA)$. The mismatch of the lattice along the CNT axis causes a $2 \%$ compression in the gold layer, while such a mismatch with the aluminum layer is negligible. Along the perpendicular direction $(z)$ to the two-dimensional slab, the compound of the nanotube and the metal layers is separated from its replica by a large vacuum region $(\approx 10 \AA)$. Figure $1(\mathrm{a})$ is a cross-sectional side view of the $\mathrm{CNT}$ on the $\mathrm{Al}(111)$ surface. A top view of the $(10,0)$ nanotube on the oxidated $\mathrm{Al}(111)$ is shown in Fig. 1(b). For visual clarity, the CNT in Fig. 1(b) is drawn shifted along the axial direction to avoid overlap with the metal layers beneath. Overall structure for the gold case is nearly the same with Fig. 1(a) (not shown here). To construct a model geometry for the oxidated surface, we assume that all the fcc hollow sites are occupied by oxygen atoms, as shown in Fig. 1(b). The oxidation of aluminum has been discussed in the literature, ${ }^{22,23}$ and the adsorption on the fcc sites has been known as the most stable. ${ }^{24}$

Projected densities of states (PDOS) for the $(10,0)$ nanotube on the $\mathrm{Al}(111)$, and $\mathrm{Au}(111)$, and oxidated $\mathrm{Al}(111)$ surfaces are shown in Figs. 1(c), 1(d), and 1(e), respectively. We observe that the Fermi level of the aluminum (gold) surface sits at the conduction (valence) band edge of the semiconducting $(10,0)$ nanotube. A previous work ${ }^{25}$ investigated the $(8,0)$ nanotube in contact with $\mathrm{Au}(100)$ surface, and reported
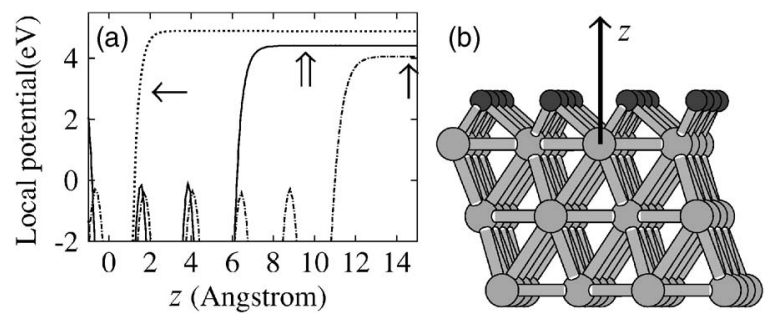

FIG. 2. (a) Electrostatic potentials (eV) of the clean and oxidated $\mathrm{Al}(111)$ surfaces are averaged in the $x y$ plane and plotted along the direction $(z)$ perpendicular to the surface. The upward single arrow (dot-dashed line) indicates the potential for the case with clean $\mathrm{Al}(111)$ surface. The leftward single arrow (dotted line) denotes the potential when the oxygen coverage on the $\mathrm{Al}(111)$ surface is the same as that shown in Fig. 1(b), while the potential when the oxygen coverage is half of that shown in Fig. 1(b) is indicated by the upward double arrow (solid line). (b) The side view of the oxidated $\mathrm{Al}(111)$ surface. A symmetric slab composed of six aluminum layers with or without oxygen on the outmost layers is used in the calculations, but only the half of the slab is shown in (b). In (a), $z$ measures the distance from the topmost aluminum layer, as indicated by the arrows in (b). However, note that the potential lines in (a) indicated by the upward double arrow and upward single arrow are shifted right by 5 and $10 \AA$ for visual clarity.

a similar result to ours. When the CNT is in contact with the oxidated $\mathrm{Al}(111)$ surface, as shown in Fig. 1(b), the Fermi level shifts downward by about $0.5 \mathrm{eV}$ and is then aligned at the valence band edge of the nanotube, which is shown in Fig. 1(e). This result may suggest that the oxidated Al surfaces have a work function similar to that of the clean gold surfaces. The work function of the gold surfaces is known to be larger than that of the aluminum surfaces by about 0.8 eV. ${ }^{26}$ In Figs. 1(c) and 1(d) we note small traces of density of states within the band gap of the nanotube. These obviously come from the metallic states. In the calculation of the PDOS, we projected Kohn-Sham eigenstates into the Wigner-Seitz spheres whose radii are $2 \AA$ for the metal atom (Al and $\mathrm{Au}$ ) and $1 \AA$ for the carbon atom, respectively. Since the nearest metal-carbon distance in the case of Fig. 1(a) is about $3.2 \AA$, part of metallic states could be overlapped in the projection into carbon atoms. However, in Fig. 1(e), such a trace of density of states in the midgap disappears as the metal-carbon distance increases, due to the intervening oxygen atoms.

For more detailed investigation, we calculate the work function of $\mathrm{Au}(111)$ and $\mathrm{Al}(111)$ surfaces and its increment upon the oxygen adsorption. A symmetric slab composed of six metal layers is positioned in the supercell with a large vacuum region $(\approx 10 \AA)$. The oxidated $\mathrm{Al}(111)$ surface with the same coverage as shown in Fig. 1(b) is investigated. The single electron potential, without nonlocal terms, is then averaged in the $x y$ plane and plotted along the $z$ direction, as shown in Fig. 2(a), where the energy reference is the Fermi level and $z$ measures the distance from the topmost aluminum layers as in Fig. 2(b). In this calculation the work function of the $\mathrm{Au}(111)$ surface is found to be $5.28 \mathrm{eV}$, while that of the $\mathrm{Al}(111)$ is about $4.12 \mathrm{eV}$. These are largely consistent with the literature values. ${ }^{26,27}$ Interestingly, the oxidation- 


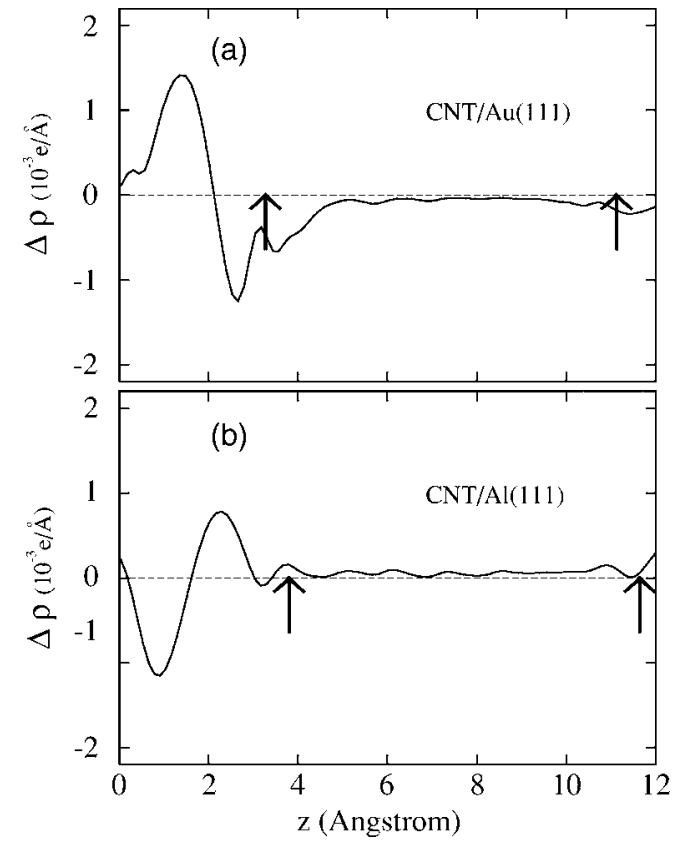

FIG. 3. The charge density differences, $\Delta \rho=\rho[\mathrm{CNT} / M(111)]$ $-\rho(\mathrm{CNT})-\rho[M(111)]$, where $M$ represents (a) Au and (b) Al slabs, respectively, are averaged in the $x y$ plane and plotted along the direction perpendicular to the surface. The $z$ measures the distance from the topmost metal layer, and arrows indicate the position of the carbon nanotube edge.

induced increment of the work function of the $\mathrm{Al}(111)$ surface is about $0.8 \mathrm{eV}$, and thus the oxidated $\mathrm{Al}(111)$ surface has a work function similar to the $\mathrm{Au}(111)$ surface, as discussed above. Indeed, the increment of the work function could depend on the coverage of the oxygen adsorbates. When the oxygen coverage is half of that for Fig. 1(b), the work function increment is also about half of the aforementioned value. When we calculate for the nanotube in contact with the oxidated $\mathrm{Al}(111)$ surface with such a half oxygen coverage, the Fermi level of the aluminum is found to be (not shown) around the midgap of the nanotube. We note that this oxygen coverage could be related to the transfer character of the CNT-FET. Derycke et al. demonstrated that an intermediate oxygen exposure to the device induces an ambipolar behavior, while higher doses of oxygen result in the unipolar $p$-type behavior. ${ }^{10}$

Despite the large work function change $(\approx 0.8 \mathrm{eV})$ upon the oxidation, the alignment of the Fermi level changes only about $0.5 \mathrm{eV}$, from the conduction band edge to the valence band edge of the semiconducting carbon nanotube, as shown in Figs. 1(c) and 1(e), respectively. Such a change for the Fermi level alignment results from the interplay between the work function changes in the metal surfaces and a small dipole field developed at the interface between the nanotube and the metal surface. In Fig. 3, the charge density difference $\Delta \rho=\rho[\mathrm{CNT} / M(111)]-\rho(\mathrm{CNT})-\rho[M(111)]$, where $M$ represents $\mathrm{Al}$ or $\mathrm{Au}$ slab, are averaged in the $x y$ plane and plotted along the perpendicular direction $(z)$ to the surface. We note a small charge depletion and accumulation in the overall nanotube region, when the $\mathrm{CNT}$ is in contact with $\mathrm{Au}(111)$ and $\mathrm{Al}(111)$, respectively. However, the more noticeable fea- ture is the charge rearrangement in the interface. The electron charge accumulates on the metal side in Fig. 3(a), while accumulating on the CNT side in Fig. 3(b). Thus the dipole field would direct from $\mathrm{Au}(111)$ to the $\mathrm{CNT}$ surface when CNT is in contact with the $\mathrm{Au}(111)$ surface, while it would from the CNT surface to the $\mathrm{Al}(111)$ surface when the CNT is on the $\mathrm{Al}(111)$ surface. This dipole field compensates the work function difference in the metal surfaces. The nanotube in contact with the oxidated $\mathrm{Al}(111)$ surface shows nearly the same features as the case of the clean $\mathrm{Au}(111)$ surface (not shown here).

For our calculations we use the periodic boundary condition, and thus the model geometries shown in Fig. 1 assume an infinitely long interface between metal and nanotube. In a realistic device structure, however, only parts of the nanotube are in contact with the metal, while the remaining part of the CNT forms a channel, connecting the source and drain electrodes. If the metal-induced gap state (MIGS) is strong at the interface in the contact region, the Schottky barrier height, that the tunneling electron experiences as it pass through from the contact into the CNT body in the channel region, may not be obtained from the Fermi level alignment, as presented in Fig. 1. However, it has been discussed that, when the nanotube is separated from the metal surface with the van der Waals distance, the MIGS decays sufficiently in the vacuum region between the metal and nanotube. ${ }^{28}$ Thus the Schottky barrier height at such a contact can be directly obtained from the Fermi level alignment in the model geometry, as presented in previous paragraphs.

Despite that our electronic structure calculations rationalize the suggestion about the oxygen effect on the Schottky barrier at the metal-nanotube contacts, the specific goldnanotube contact case, showing $n$-type transport behavior in the vacuum, ${ }^{10}$ is not fully resolved. Since the gold surface has a high work function, the gold-nanotube contact should have resulted in the $p$-type Schottky contact rather than $n$ type. Especially, according to the suggestions by Leonard and Tersoff, the Schottky barrier between the metal and nanotube does not so much depend on the strength of the interface coupling at the nanosized contacts. ${ }^{29}$ In this regard, Yamada explained that the Schottky barrier for hole transport could be lowered by the intervening oxygen molecules at the gold-nanotube contact. ${ }^{30}$ Shan et al. showed that the Fermi level alignment at the contact between the nanotube and the large work function metal could depend on the interfacial distance. ${ }^{31}$ Nevertheless, we note that our ab initio results, showing the Fermi level alignment at the conduction band edge (the clean $\mathrm{Al}$ case) or at the valence band edge (the clean $\mathrm{Au}$ and oxidated $\mathrm{Al}$ cases), could be a generic model explaining the oxygen-induced conversion from $n$-type to $p$-type behaviors of the FET.

The oxygen adsorption not only affects the Schottky barrier, but the tunneling barrier at the metal and nanotube contact as well. When the nanotube is separated from metal surface with the van der Waals distance, electrons experience a vacuum barrier as they tunnel from metal to the nanotube. A part of the contact resistance can be attributed to such a tunneling barrier. ${ }^{32}$ In order to investigate the effect of oxygen adsorption on the tunneling barrier, we place the metallic $(5,5)$ nanotube on the clean and oxidated $\mathrm{Al}(111)$ surfaces, 


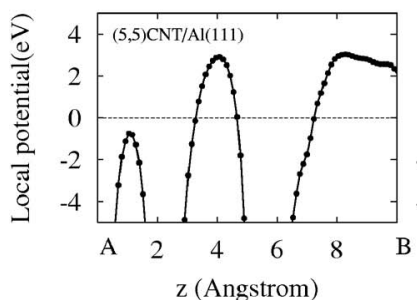

(a)

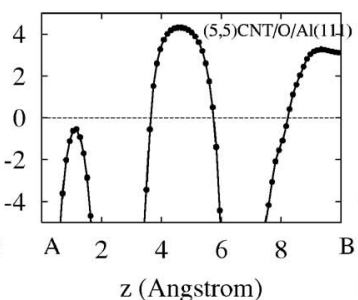

(b)

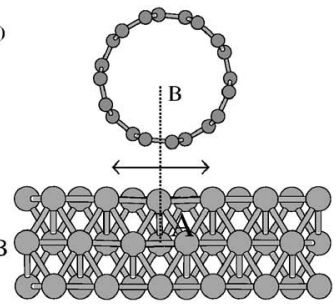

(c)
FIG. 4. Averaged electrostatic potentials for the $(5,5)$ nanotube on (a) the $\mathrm{Al}(111)$ surface and (b) the oxidated $\mathrm{Al}(111)$ surface are plotted with respect to the Fermi level. The relaxed geometry for $(5,5)$ nanotube on the $\mathrm{Al}(111)$ surface is shown in (c). The potentials in (a) and (b) are averaged over the strip whose width is indicated by the horizontal arrows in (c). respectively. We assume that the oxygen coverage on $\mathrm{Al}(111)$ is the same as that shown in Fig. 1(b). The self-consistent potential in a single-particle Kohn-Sham equation, except the nonlocal component, is plotted along the line acrossing the nearest contact point between metal and the nanotube, as denoted by the line $\mathrm{AB}$ in Fig. 4(c). Potential energies in Figs. 4(a) and 4(b) are referenced to the Fermi levels. The first dips at around $z=2.3 \AA$ in Figs. 4(a) and 4(b) correspond to the topmost $\mathrm{Al}$ layers. The nanotube wall begins at the second dips which is at about $z=5.3 \AA$ in Fig. 4(a) and $z=6.4 \AA$ in Fig. 4(b), respectively. Shown in Fig. 4(c) is the optimized geometry for the $(5,5)$ nanotube on the $\mathrm{Al}(111)$ surface. The potentials in Figs. 4(a) and 4(b) are averaged over the strip whose width is indicated by the horizontal arrow in Fig. 4(c). The barrier height is about 2.92 and 4.33 $\mathrm{eV}$ in Figs. 4(a) and 4(b), respectively, and thus we have a substantial increase in the tunneling barrier upon the oxidation. The barrier heights along the line AB without the average are almost the same as the aforementioned values. Using the WKB approximation we calculate the transmission probability ${ }^{33} T=e^{-2 \sqrt{2 m / \hbar^{2}}} \int \sqrt{V(z)-\varepsilon_{f}} d z$ across the vacuum barriers $[3.2 \leqslant z \leqslant 4.8$ for Fig. $4(\mathrm{a})$, and $3.7 \leqslant z \leqslant 5.8$ for Fig. 4(b)]. Through the numerical integration, we find that the transmission probability for Fig. 4(b) is 0.15 times that for Fig. 4(a). This implies that the contamination of one oxygen monolayer could result in an order of magnitude smaller current or larger contact resistance.

\section{SUMMARY}

We investigated the Schottky barrier at metal-CNT contacts and its variation upon oxidation, using $a b$ initio electronic structure calculations. We found that the Fermi level of gold is aligned at the valence band edge, while that of the aluminum sits at the conduction band edge of the semiconducting nanotube. The work function increment is found to be about $1 \mathrm{eV}$ upon the oxidation of the $\mathrm{Al}(111)$ layer, which causes the Fermi level to be aligned at the valence band edge of the nanotube. Our $a b$ initio calculations provided a model, in terms of the electronic structure, to explain the conversion from $n$-type to $p$-type behaviors of the CNT-FET upon oxygen adsorptions. We also investigated the effect of oxygen adsorption on the tunneling barrier between metal and nanotube.

\section{ACKNOWLEDGMENT}

We acknowledges support from the KOSEF through the Center for Nanotubes and Nanostructured Composites.
*Email address: noejung@dku.edu

${ }^{\dagger}$ Email address: hong@sejong.ac.kr

${ }^{1}$ S. Iijima, Nature (London) 354, 56 (1991).

${ }^{2}$ S. Frank, P. Poncharal, Z. L. Wang, and W. A. de Heer, Science 280, 1744 (1998).

${ }^{3}$ A. Javey, H. Kim, M. Brink, Q. Wang, A. Ural, J. Guo, P. McIntyre, P. McEuen, M. Lundstrom, and H. Dai, Nat. Mater. 1, 241 (2002).

${ }^{4}$ A. Javey, J. Guo, Q. Wang, M. Lundstrom, and H. Dai, Nature (London) 424, 654 (2002).

${ }^{5}$ S. J. Tans, A. R. M. Verschueren, and C. Dekker, Nature (London) 393, 49 (1998).

${ }^{6}$ S. G. Rao, L. Huang, W. Setyawan, and S. Hong, Nature (London) 425, 36 (2003).

${ }^{7}$ S. Heinze, J. Tersoff, and Ph. Avouris, Appl. Phys. Lett. 83, 5038 (2003).

${ }^{8}$ J. Appenzeller, J. Knoch, V. Derycke, R. Martel, S. Wind, and Ph. Avouris, Phys. Rev. Lett. 89, 126801 (2002).

${ }^{9}$ M. Freitag, M. Radosavljevic, Y. Zhou, A. T. Johnson, and W. F. Smith, Appl. Phys. Lett. 79, 3326 (2001).
${ }^{10}$ V. Derycke, R. Martel, J. Appenzeller, and Ph. Avouris, Appl. Phys. Lett. 80, 2773 (2002).

${ }^{11}$ S. Heinze, J. Tersoff, R. Martel, V. Derycke, J. Appenzeller, and Ph. Avouris, Phys. Rev. Lett. 89, 106801 (2002).

${ }^{12}$ P. G. Collins, K. Bradley, M. Ishigami, and Z. Zettl, Science 287, 1801 (2001).

${ }^{13}$ G. U. Sumanasekera, C. Adu, S. Fang, and P. C. Eklund, Phys. Rev. Lett. 85, 1096 (2000).

${ }^{14}$ S.-H. Jhi, S. G. Louie, and M. L. Cohen, Phys. Rev. Lett. 85, 1710 (2000).

${ }^{15}$ H. Ulbricht, G. Moos, and T. Hertel, Phys. Rev. B 66, 075404 (2002).

${ }^{16}$ P. Giannozzi, R. Car, and G. Scoles, J. Chem. Phys. 118, 1003 (2003).

${ }^{17}$ S. Dag, O. Gülseren, T. Yildirim, and S. Ciraci, Phys. Rev. B 67, 165424 (2003).

${ }^{18}$ G. Kresse and J. Hafner, Phys. Rev. B 47, R558 (1993); 49, 14251 (1994).

${ }^{19}$ G. Kresse and J. Furthmüller, Phys. Rev. B 54, 11169 (1996); Comput. Mater. Sci. 6, 15 (1996). 
${ }^{20}$ J. P. Perdew, K. Burke, and M. Ernzerhof, Phys. Rev. Lett. 77, 3865 (1996).

${ }^{21}$ G. Kresse and D. Joubert, Phys. Rev. B 59, 1758 (1999).

${ }^{22}$ K. Honkala and K. Laasonen, Phys. Rev. Lett. 84, 705 (2000).

${ }^{23}$ T. Sasaki and T. Ohno, Phys. Rev. B 60, 7824 (1999).

${ }^{24}$ J. Jacobsen, B. Hammer, K. W. Jacobsen, and J. K. Nørskov, Phys. Rev. B 52, 14954 (1995).

${ }^{25}$ S. Dag, O. Gülseren, S. Ciraci, and T. Yildirim, Appl. Phys. Lett. 83, 3180 (2003).

${ }^{26}$ H. B. Michaelson, J. Appl. Phys. 48, 4729 (1977).

${ }^{27}$ C. J. Fall, N. Binggeli, and A. Baldereschi, Phys. Rev. B 58,
R7544 (1998).

${ }^{28}$ Y. Xue and S. Datta, Phys. Rev. Lett. 83, 4844 (1999).

${ }^{29}$ F. Léonard and J. Tersoff, Phys. Rev. Lett. 84, 4693 (2000).

${ }^{30}$ T. Yamada, Phys. Rev. B 69, 125408 (2004).

${ }^{31}$ B. Shan and K. Cho, Phys. Rev. B 70, 233405 (2004).

${ }^{32}$ M. S. Fuhrer, J. Nygøard, L. Shin, M. Forero, Y.-G. Yoon, M. S. C. Mazzoni, H. J. Choi, J. Ihm, S. G. Louie, A. Zettl, and P. L. McEuen, Science 288, 494 (2000).

${ }^{33}$ R. L. Liboff, Introductory Quantum Mechanics, 2nd ed. (Addison-Wesley, New York, 1992). 